\title{
CALIDAD Y EXPERIENCIA DOCENTE EN EDUCACIÓN SUPERIOR
}

\author{
María Isabel Lafuente Guantes ${ }^{1}$ \\ Universidad de León - España \\ Grupo de Investigación HISULA \\ milafg@unileon.es
}

Recepción: 30/05/2010

Evaluación: 02/07/2010

Aceptación: 14/11/2010

Artículo de Reflexión

\section{RESUMEN}

En este trabajo se considera la forma de entender la calidad en la experiencia docente en educación superior a partir de la práctica del profesorado, y en relación con las orientaciones educativas hoy al uso. Se analiza en tres trabajos: las nociones en que se basa esta experiencia, su valor en el proceso docente, qué condiciones se requieren para lograr que la creencia en la calidad alcance su efectuación

Palabras clave: Calidad, Experiencia Docente, Creencia, Conocimiento, Organización Del Conocimiento.

\footnotetext{
1 Doctora en Filosofía y Letras por la Universidad de Oviedo. Catedrática de Universidad del Área Filosofía. Licenciatura, Universidad Complutense de Madrid, dos sexenios de investigación y seis quinquenios docentes. Actualmente es miembro del Grupo de Investigación Historia y Prospectiva de la Universidad Latinoamericana HISULA.
}

\begin{tabular}{l|l} 
Rev. hist. edu. latinoam. Vol. 15. Año 2010, pp. $177-196$ \\
\hline &
\end{tabular}




\title{
TEACHER'S QUALITYAND EXPERIENCE IN HIGHEDUCATION
}

\author{
María Isabel Lafuente Guantes \\ Universidad de León - España \\ HISULA Research Group \\ milafg@unileon.es
}

\begin{abstract}
In this work it is considered the way of understanding the quality in the educational experience in the university from the practice of its professionals, and in relation with the educational current guidelines. There is analyzed in three works: the main ideas in this experience is based, the value on the educational process, what conditions are needed to achieve quality and what its accomplishment demands.
\end{abstract}

Key words: Quality, Educational Experience, Belief, Knowledge, Organization of the Knowledge. 


\section{INTRODUCCIÓN}

Últimamente proliferan los estudios que atienden a la calidad en la práctica del profesor universitario, seguramente impulsados por factores tan importantes como las modificaciones que en la educación superior están produciendo los cambios en la legislación, y que aún se desearía que fueran mayores dado el excesivo fracaso del alumnado en las carreras universitarias. Estos estudios son multivalentes, unos se preocupan por saber sobre la calidad basándose en narraciones realizadas por los mismos profesores, otros se apoyan en el estudio de la legislación, otros en la aplicación de modelos elaborados por profesionales de la educación, pero todos intentan mostrar de forma objetiva cuál es el estado de la educación superior, general o según especializaciones, y qué factores permiten calificar de excelente la práctica docente.

La forma de proceder en estos estudios es diversa: algunos parten de una consideración general relativa a cómo entender hoy la universidad, otros comienzan preguntándose a qué responden las legislaciones, otros consideran fundamental partir de cómo entender la experiencia, y otros intentan precisar qué nociones fundamentan una práctica docente de calidad, cómo aprecian los profesores su experiencia docente, y cómo orientarla de forma satisfactoria. En este último caso, o bien se considera una noción como la necesaria para orientar la experiencia del profesor, o bien se entiende que son varias nociones en las que reside la calidad, siendo las más frecuentes: docencia, investigación y gestión, que se hacen depender de varios factores, por ejemplo, tiempo, especialidad, objetivos, expectativas, etc.

Como en este trabajo nos vamos a limitar a considerar la forma de orientar hacia la calidad la experiencia del profesor universitario y, por tanto, a saber qué requisito o requisitos son necesarios para que su práctica sea de calidad e incida de esta forma en el proceso docente, nuestro objetivo se concreta a intentar saber, a partir del análisis de algunos trabajos sobre el tema, de qué forma entender esta experiencia, en qué factores reside su calidad, y cómo pueden incorporarse positivamente al proceso educativo.

Situándonos en este campo, vamos a comenzar por preguntarnos cómo entender hoy la experiencia docente, pues ciertamente si hace veinte años esta noción sólo adquiría validez cuando se pretendía saber sobre las reivindicaciones de colectivos de profesionales docentes y sus posibilidades de realización (estabilidad, mejoras económicas, etc., en las que muchas veces se enfrentaban unos colectivos a otros), hoy adquiere su máxima importancia en el examen de las condiciones generales que afectan a la forma en que el profesor influye en 
el proceso educativo, por lo que hoy constituye una de las nociones que guían los estudios sobre las prácticas de los profesores en tanto que cooperan y convergen en la búsqueda del mejor y mayor éxito del proceso.

Hasta el momento actual los trabajos que se están realizando por los profesionales de la educación sobre el tema, están influyendo muy poco y tan sólo en las políticas educativas, de lo que resulta que en las prácticas docentes su influencia es sólo formal, y casi nula. Considero que este hecho se debe a que no se está produciendo una unidad suficiente de análisis y conclusiones que permitan evaluar y valorar las necesidades de variación de la relación enseñanzaaprendizaje (y de investigación-gestión), y tomar conciencia al profesorado de aquellas posibilidades existentes hoy, cuya práctica puede lograr un proceso docente más simple y más efectivo.

En tanto lo que sea necesario aportar de forma positiva a las prácticas docentes tiene que nacer de las aportaciones que resulten de la experiencia profesional, vamos a partir de analizar cómo se está entendiendo ésta, para saber si es necesario introducir variaciones en sus procedimientos, y cuáles son éstas. La generalidad de los trabajos que se realizan sobre el tema se basan en cuestionarios dirigidos a los profesores que tienen que manifestar en sus respuestas la forma en la que entienden su profesión, proceden en ella y la valoran. Evidentemente estos trabajos se apoyan en la experiencia que el profesor posee y recoge de su práctica, permitiendo entender la forma en que ésta se concibe. Lograr establecer mediante las respuestas qué es necesario para el mejor desarrollo de la tarea docente, es lo que puede permitir saber sobre la forma de incidir para lograr un proceso docente mejor cualificado, rentable y con logros más satisfactorios.

\section{Las dos Orientaciones Educativas}

Vamos a partir de la idea de que trabajar en Ciencias de la Educación, puede tener distintas orientaciones, y que las dos más significativas son las que señala J. Larrosa en su conferencia Algunas notas sobre la experiencia y sus lenguajes, en donde paralelamente a la distinción ciencia /tecnología y teoría/práctica, señala: "Para los positivistas, la educación es una ciencia aplicada. Para los así llamados críticos, la educación es una praxis reflexiva." ${ }^{2}$ J. Larrosa considera que la experiencia es siempre fugaz, impura, confusa, mutable, ligada a situaciones

\footnotetext{
2 La conferencia de Larrosa que cito se halla en Web la siguiente dirección: www.mc.gov.ar/cumform/publica/oei/oei_20031128/potencia-larrosa.pdf Esta página fue consultada el día 25 de abril de 2007.
} 
particulares y contextuales, de forma que la ciencia tiene que convertirla en experimento para objetivarla, homogeneizarla y universalizarla. A partir de esta consideración, este autor sostiene que darle todo su valor a la experiencia supone: separarla de todo experimento, quitarle todo dogmatismo, es decir, toda pretensión de autoridad, aislarla de la práctica, y quitarle la condición de fetiche, o de imperativo, pues a lo que realmente se halla ligada es a la pasión, al sentimiento, y lo que expresa es la condición de apertura, la capacidad de mostrar que las cosas tienen un sentido, el que corresponde a mis propias vivencias. Estas consideraciones le valen para caracterizar la existencia en la forma siguiente:

Personalmente, he intentado hacer sonar la palabra experiencia cerca de la palabra vida o, mejor, de un modo más preciso, cerca de la palabra existencia. La experiencia sería el modo de habitar el mundo de un ser que existe, de un ser que no tiene otro ser, otra esencia que su propia existencia. ${ }^{3}$

Este autor, cercano a las tesis de W. Benjamín, reivindica como orientación propia del contexto educativo el relato, al que entiende como facultad de intercambiar experiencias, lo que no se halla muy lejos de trabajos como el de A. Gewere ${ }^{4}$ cuando entiende, en su trabajo sobre la identidad del profesorado, que la forma de acceso al conocimiento de los modos en que se configura la subjetividad tiene que ser, siguiendo a P. Ricoeur, la narración, y su objetivo básico la descripción y la comprensión.

Las consideraciones de J. Larrosa, como las de A. Gewere, son propias de una de las formas más importantes del trabajo actual en las ciencias humanas: la metodología cualitativa, que constituye hoy una orientación fundamental en educación, y cuya base filosófica es la hermenéutica de la experiencia, que comienza a gestarse con Dilthey, se desarrolla en forma de metafísica en Heidegger, y es continuada por Gadamer, Habermas etc. Dilthey pretendió que la hermenéutica, cuya base es la comprensión, se constituyera en la metodología propia de las Ciencias Humanas. La consideración de la hermenéutica por Gadamer, si bien es cierto que en Verdad y Método hay un momento dubitativo en que intenta mostrar su necesidad para las ciencias en general, ha seguido la vía que marcó Heidegger al entenderla como la forma de comprensión de lo humano, cuyo contexto es la experiencia general gestada en y por el lenguaje, y sin embargo ya se considera imprescindible por muchos teóricos de la ciencia como fundamento de toda metodología científica.

\footnotetext{
3 Ibídem., p.15.

4 GEWERE, A. (2001): "Identidad profesional y trayectoria en la universidad", en: Profesorado. Revista de Vitae y Formación de Profesorado. Año/vol. 5. nº 002. Granada, Universidad de Granada, p. 67.
} 
En una situación como la actual, vitalmente crítica, debida a que el proceder político-científico ha conducido a un abismo nihilista, a una perdida del sentido vital, se intenta recuperar y fortalecer la confianza en cada acción humana desde la hermenéutica como el lugar en que tiene lugar la producción comprensiva de sentido e intersubjetividad. En el ámbito del conocimiento crítico se debe a los estudios de Habermas la exigencia de atender a la hermenéutica en el contexto general de la cientificidad, pues si bien la distinción entre acción instrumental y acción comunicativa puede llevar a pensar que la hermenéutica solo sirve de base a esta última, como Habermas la considera base del proceso cognoscitivo humano su problemática, si bien constituye una exigencia previa a la producción de conocimiento científico, se halla involucrada necesariamente en él. Actualmente esta orientación concretada en la metodología cualitativa ha hecho pasar a primer plano las historias de vida, la narración de vivencias, el diálogo sobre las consideraciones subjetivas de las situaciones, en otras palabras el conocimiento de lo que hoy se conocen como los rituales de vida individuales, que se entienden como las comprensiones vitales en las que tiene que apoyarse el conocimiento reflexivo. La base de la experiencia vital, de la historia efectual, es la negación determinada, que como es sabido se muestra en la dialéctica negativa de Adorno como negación de la negación, que en su raíz supone posible (al menos para la humanidad) lograr una idea positiva mediante la creación de nuevos significados, logrados por medio de narraciones o formas poéticas, que permiten la apertura a nuevo horizonte. Este contexto cualitativo tiene la propiedad de constituir la metáfora como un tipo de razón sobre las cosas.

La otra orientación en educación, la que la entiende como productora de conocimientos-científico educativos, por tanto como Ciencias de la Educación, parte de la unidad en la investigación de la metodología cualitativa y la cuantitativa, y del supuesto de que el trabajo en este ámbito no puede consistir en la creación de adornos literarios sobre las concepciones y prácticas desarrolladas en el proceso docente, sino que es necesario producir un conocimiento válido y fiable encaminado a producir las mejores orientaciones educativo-culturales y la mejor forma de proceder en el desarrollo de la práctica docente e investigadora. En este caso, considero que la situación general actual necesita mejores conocimientos de los problemas epistemológicos generales, así como de los que suscitan las aplicaciones al campo determinado de estudio.

\section{La noción de experiencia docente relativamente las orientaciones educativas}

Epistemológicamente no es fácil dilucidar cuál es el lugar y el valor de la experiencia. Es evidente que la noción de experiencia nos sitúa en un análisis 
empírico de la situación, pues históricamente constituye el principio de la metodología empirista. Pero, si nos preocupamos por saber cómo ha sido su consideración en la historia del conocimiento, podemos observar que en ésta se manifiesta la misma condición que reflejan los estudios actuales. En efecto, en el siglo XVI se contrapone experiencia a principios, y en el siglo XVIII, en plena Ilustración, se opone experiencia a construcciones a priori. En ambos casos el problema que soportan los estudios ligados a la experiencia es el escepticismo (su última forma es el nihilismo), pues los datos empíricos por sí mismos no pueden dar lugar a constituir una totalización consistente, única forma de poder dar respuesta a los problemas de identidad y causalidad: ¿qué es esto?, ¿de qué depende esto?, preguntas que sin las que es imposible el paso a saber cómo es posible variar la situación.

Uno de los problemas relativos a la noción de experiencia, pues afecta al valor del conocimiento que se basa en ella, es la distinción entre experiencia vulgar y experiencia científica. La primera se entiende como una experiencia ordinaria, común, que puede lograr, todo lo más, saber sobre las opiniones más generalizadas, la segunda como una ordenación de los datos según técnicas expuestas, y acompañada de una reflexión crítica sobre el valor cognoscitivo y el fundamento de la certeza que la construcción realizada produce.

Esta distinción puede llevar a entender que la experiencia vulgar está afectada de escepticismo, pero no la científica, ahora bien, también puede conducir, como hace Popper, a sostener que el conocimiento científico tampoco pasa de ser una conjetura. Por ello, eliminar el escepticismo supone considerar que, lo que se llama experiencia vulgar es la experiencia individual, y que la noción de experiencia tiene que considerarse como un concepto válido tanto para permitir atender a las creencias individuales (experiencia individual), como a los modelos posibles ante determinadas situaciones (experiencia objetiva), lo que supone admitir que existe un marco común a ambas, experiencia general, que permite ver la experiencia científica como la perfección de la experiencia individual (vulgar) y como la meta a que ésta tiende. ${ }^{5}$

En este último caso, los elementos cualitativos de la experiencia, nacidos de las narraciones individuales, tienen que considerarse con un valor propio (aunque puedan someterse en su mayor parte a cuantificación), pues científicamente no

\footnotetext{
5 La idea de que la experiencia científica es la perfección de la vulgar se haya tanto en la consideración epistemológica de M. Bunge como en la K. Popper (este habla de sentido común), pero mientras el primero entiende que es posible alcanzar un conocimiento científico válido y verdadero, el segundo se mantiene en una consideración escéptica del conocimiento, no tanto por entender que está aquejado de incertidumbre, como por sostener que nunca puede pasar de ser una conjetura.
} 
sólo tiene importancia considerar los problemas, su estado y su solución, relativamente a la cuantificación de los datos, sino que también es necesario contar con las posibilidades sinergéticas (emergentes, en la terminología de Bunge) que aporta lo cualitativo, y con la variaciones en la concepción y en la práctica que puede comportar la apertura de sentido. Por, ejemplo, al considerar el tema de la selección del profesorado, no sólo tendrán importancia resultados como el número de plazas cubiertas, en las distintas titulaciones y en un determinado período de tiempo, el nivel de esas plazas y su coste económico para la inversión en educación, sino también las condiciones cualitativas que suponen los rituales en que se llevan a cabo esas dotaciones, en cuanto entronizan unas determinadas formas de proceder que pasan a considerarse las idóneas en un sistema, pero que pueden repercutir muy negativamente en la realidad cotidiana del proceso educativo. Ahora bien, contar con análisis cualitativos supone introducir factores que pueden llevar la investigación hasta niveles de complejidad muy altos, lo que exige, si no se quiere que el análisis termine en formulaciones ideológicas, controlar las relaciones entre los elementos cuantificables y su relación con los cualitativos, de forma que no solo las hipótesis sean verificadas de forma fiable, sino que los resultados adquieran un valor interpretativo bien consolidado.

Nociones de experiencia como las que se encuentran en enciclopedias, o las que se usan sin conocimiento de los problemas epistemológicos generales, ni de los nacidos de la aplicación en el campo concreto que se trata de considerar, no son suficientes para realizar un análisis con validez. Entre las múltiples caracterizaciones que se encuentran hoy de la experiencia, una de enfoque cognitivo, particularmente de gestión del conocimiento, pero en la que se muestra la cuestión planteada, es la siguiente:

\begin{abstract}
La experiencia puede ser vista como un catálogo, un conjunto de modelos de respuesta ante determinadas situaciones y estímulos. Estas respuestas vienen condicionadas por nuestras actitudes emocionales, nuestras representaciones mentales personales y nuestros procesos conscientes de decisión...Las actitudes emocionales son totalmente personales y no pueden ser transferidas por procesos cognitivos... Cada uno forja los suyos propios a través de sus vivencias...Estas representaciones son susceptibles de ser modificadas y reestructuradas... ${ }^{6}$
\end{abstract}

Esta forma de explicar la experiencia pone de relieve que el modelo o conjunto de modelos de conducta que permiten entenderla, tienen su base en la experiencia personal, es decir, en las repuestas de los sujetos, pero no establece

\footnotetext{
6 Esta explicación de la noción de experiencia se halla en: http://www.wikilearning.com/
} que_es_la_experiencia-wkccp-16284-4.htm. ultima actualización el 24 de abril de 2007. 
ningún criterio que permita considerar su valor científico. Para dar cuenta del carácter científico de la experiencia, es necesario que se precisen los principios o hipótesis de que depende la construcción de estos modelos, principios que tienen que ser debidamente contrastados, pues de otra forma la interpretación en lugar de adquirir carácter científico, lo adquiere ideológico.

Esto sucede, entre otros casos, cuando se usan clasificaciones establecidas, interpretando las respuestas de los sujetos de forma que encajen en los modelos, o no se enuncia qué criterios se usan para establecer una clasificación, o no son expuestos de forma apropiada, o no se realiza la interpretación de forma crítica. Los trabajos cuyos principios, en los que reside la capacidad interpretativa del modelo, no son explicitados de forma que tampoco es posible la formulación de hipótesis que puedan ser contrastadas, no es que carezcan de valor en el conjunto de las investigaciones sobre el tema, sino que tienen que considerarse, en el mejor de los casos, como opiniones doctas, pero como opiniones, sobre las que ulteriormente habrá que realizar trabajos que ofrezcan mayor fiabilidad en el tratamiento epistemológico de los datos.

Realmente, cuando se trabaja sobre la experiencia del profesorado es necesario poner en relación ambas formas de experiencia, explicitar tanto los principios que permiten realizar un modelo o modelos cuantificables, como aquellos elementos cualitativos que las técnicas empleadas no permiten establecer de forma cuantitativa, pero que permiten una interpretación sobre la situación analizada que muestre variaciones posibles en el modelo cuantitativo y, finalmente, que aportación supone dicha investigación respecto de la situación actual.

\section{Principios conformadores de la experiencia docente del profesor universitario: creencia, conocimiento y competencia}

Vamos a considerar tres investigaciones de las que nos valdremos para saber como se entiende hoy la experiencia del profesor analizando: qué aportaciones realizan y cuáles pueden derivarse de ellas. Es importante señalar que en dos de estas investigaciones la noción de creencia puede entenderse perfectamente como el criterio básico conformador de la experiencia del profesorado; que en cada una se atiende de forma diferente a esta noción, pero que siempre se expone el concepto o conceptos que se van a seguir en la investigación justificando de forma expresa la razón por la que consideran la creencia como principio el conformador. La tercera investigación considera la experiencia en relación con la antigüedad, (tiempo), factor que se considera es el que permite valorar los otros temas, y relativamente al cuál se van a valorar creencias, motivaciones y opiniones del profesorado encuestado. 
Los tres trabajos han sido seleccionados por las siguientes razones: llevan a cabo formularios muy precisos, que permiten deslindar en las respuestas los factores cualitativos de aquellos que pueden ser cuantificados: los tres establecen con precisión los métodos usados y la forma de su aplicación; delimitan perfectamente la población investigada y establecen herramientas (instrumentos) de cuantificación de las que evalúan su fiabilidad, así como muestran con claridad qué principios son los que guían la investigación y cómo llegan a formular y contrastar las hipótesis que posibilitan.. Sin embargo, en casos puntuales, la exposición y presentación de los resultados de cada item, aunque tiene lugar de forma clara, ordenada y siguiendo los mismos criterios, no lleva a articular una explicación precisa de las contradicciones que se generan, de forma que las conclusiones parecen, al menos a primera vista, extrañas, al no resolver la oposición con el punto de vista más generalizado.

La primera investigación es la realizada en 2005 por L. Prieto Navarro, en la Universidad de Comillas de Madrid, titulada Las creencias de autoeficacia docente del profesorado universitario, ${ }^{7}$ que permite considerar la experiencia del profesor en función de sus creencias. Esta autora define las creencias como “(...) concepciones o teorías implícitas del profesorado que generan una disposición a actuar de un modo determinado". ${ }^{8}$

Justifica su estudio en cuanto las creencias pedagógicas pueden afectar a la eficacia docente del profesor, tanto en lo que respecta a motivaciones como a las estrategias didácticas, y plantea como objetivos:

1. Elaborar un cuestionario que permita conocer las creencias de autoeficacia docente del profesorado universitario.

2. Analizar las creencias de autoeficacia en las dimensiones básicas de la docencia universitaria, y su relación con el uso que hacen los profesores de las distintas estrategias didácticas.

3. Conocer si existe relación entre determinadas variables del profesorado y su percepción de autoeficacia para enseñar.

4. Analizar si existen diferencias en autoeficacia entre los profesores que se encuentran al inicio de su carrera docente y aquellos otros que llevan más de cinco años enseñando.

\footnotetext{
7 Existen en la red dos artículos en la red de la misma autora sobre el tema. Ambos son resultado de su tesis doctoral. Las direcciones web son: www.des.emerg.edu/mfp/ PrietoSintesis.pdf y www.des.emory.edu/edu/mfp/PrietoInstrument.pdf Páginas consultadas en día 25 de abril de 2007.

8 Prieto, L. (2005): op., cit., p. 2.
} 
La investigadora ha encuestado a 362 profesores de 14 universidades españolas. El análisis se mueve en el campo de la teoría cognitiva y el aprendizaje social utilizando como instrumento psicométrico una escala diseñada expresamente que titula: Escala de autoeficacia docente del profesorado universitario, cuyo coeficiente de fiabilidad estima de 0,94\% Considera la creencia en la autoeficacia no como un rasgo global de la experiencia del profesor, sino diferenciada por ámbitos concretos de la práctica docente, estos ámbitos son: planificación (objetivos de aprendizaje, selección de contenidos, decisiones), implicación (estrategias didácticas), interacción (creación de ambiente y expectativas) y evaluación (estrategias de valoración del estudiante y del trabajo realizado).

Las principales conclusiones de la autora en este caso son: 1. Que la autoeficacia del profesorado no es uniforme en los ámbitos en que se diversifica su actividad habitual; 2. Que los profesores tienden a actuar de manera coherente con lo que piensan sobre su eficacia personal; 3. Que la creencia en la autoeficacia es potenciada por la satisfacción profesional, por el grado de preparación percibida y por la responsabilidad en el aprendizaje; 4. Que influye considerablemente en su percepción los años de antigüedad en la profesión.

Las aportaciones que la investigación muestra como significativas para la relación enseñanza-aprendizaje se concretan en la afirmación de que, partiendo del supuesto de que la enseñanza de los profesores responde a sus creencias de autoeficacia en la enseñanza, una intervención en sus creencias podría instaurar, mantener, fortalecer o incluso modificar su sentimiento de autoeficacia, e introducir mejoras que se traducirían, previsiblemente, en una docencia universitaria más eficaz, orientada a favorecer la calidad del aprendizaje. La intervención podría dirigirse a cualquiera de las dimensiones, pero los resultados apuntan a que el ámbito de la evaluación es el que necesita ser fortalecido. Por otra parte, abre un interrogante fundamental: ¿tienen los profesores información para valorar su eficacia docente?, pues si la creencia en la autoeficacia puede mejorar el aprendizaje, la obtención de información por los profesores de las distintas fuentes disponibles de eficacia, podría contribuir a favorecer esta creencia. La conclusión general estima que sería necesaria una formación pedagógica del profesorado en este sentido.

La segunda investigación ha sido realizada en el área de didáctica de las matemáticas por dos autoras M. Moreno y C. Moreno, de la Universidad de Lleida (Cataluña, España), cuyo título es Concepciones y creencias de los profesores universitarios de matemáticas acerca de la enseñanza de las 
ecuaciones diferenciales. ${ }^{9}$ La investigación, cifra la experiencia del profesorado no sólo en creencias, sino también en concepciones, y consecuentemente introduce una distinción fundamental entre ambas definiendo las primeras como sigue:

Las creencias son conocimientos subjetivos, poco elaborados, generados a nivel particular por cada individuo para explicarse y justificar muchas de las decisiones y actuaciones personales y profesionales vividas. Las creencias no se fundamentan sobre la racionalidad, sino más bien sobre los sentimientos, las experiencias y la ausencia de conocimientos específicos del tema con el que se relacionan, lo que las hace ser muy consistentes y duraderas para cada individuo. ${ }^{10}$

Las autoras señalan tres tipos de creencias del profesorado: creencias sobre la enseñanza, creencias institucionales, creencias sobre el aprendizaje.

\section{Definen la noción de concepción como sigue:}

Las concepciones son organizadores implícitos de conceptos, de naturaleza esencialmente cognitiva y que incluyen creencias, significados, conceptos, proposiciones, reglas, imágenes mentales, preferencias, etc., que influyen en lo que se percibe y en los procesos de razonamiento que se realizan. El carácter subjetivo es menor en cuanto se apoyan sobre un sustrato filosófico que describe la naturaleza de los objetos matemáticos. ${ }^{11}$

La investigación se realiza en base a los cuestionarios realizados a seis profesores de matemáticas de universidad. Las autoras aplican el término creencia al caso de la experiencia de los profesores en el proceso de enseñanza y aprendizaje del que no poseen conocimientos autorizados que les permitan formar concepciones; mientras que aplican el término concepción a la experiencia del profesor en la materia de la que experto (matemáticas), puesto que les permite tener una idea sobre la naturaleza del objeto de estudio y encuadrarla en una posición histórico-filosófica concreta.

El carácter de la metodología de investigación empleada es: cualitativo, generativo, constructivo, exploratorio, descriptivo y explicativo. El análisis se realiza en dos niveles, uno global que se efectúa mediante la puesta en relación de las formas conceptuales en una tabla de doble entrada, cuya pretensión es

\footnotetext{
9 Este artículo se halla publicado en: Enseñanza de las ciencias. Vol. 21, $\mathrm{n}^{\mathrm{o}}$ 2, junio 2003. ISSN:0212-4521. Institut de Ciències de l'Educació de la Universitat Autónoma de Barcelona. Vicerrectora d'Investigació de la Universitat de Valencia, pp. 265-280.

${ }^{10}$ Ibídem., p. 267.

${ }^{11}$ Ibídem., p. 267.
} 
lograr una visión general de las concepciones y creencias, y un análisis particular que se lleva a cabo en función de una lista de descriptores, que cualitativamente sirve también para mostrar las coherencias e incoherencias existentes entre los grupos o modelos a que da lugar el análisis general. El objetivo final de la investigación es la articulación de concepciones, prácticas y creencias

Se considera mucho más fácil el análisis de las creencias, dado que se apoyan en el desconocimiento, mientras que el análisis de las competencias se considera más difícil por estar ligado a una experiencia profesional. El problema de las competencias no está claramente definido en el trabajo, pero a partir de él se puede considerar unido al conocimiento de la materia, pero no directamente, sino mediado por los valores culturales propios de la sociedad occidental, pues estos han condicionado la relación que los matemáticos han establecido entre ellos y las matemáticas. De esta forma la concepción se concibe como la postura adoptada en el conjunto de unos conocimientos mediados por valores culturales que establecen aquello que es competencia. Los valores que se expresan son: racionalismo, objetivismo, control, progreso, aperturismo y misterio. Con el fin de ordenar las concepciones y estilos docentes se parte de modelos considerados fundamentales en la concepción, de las matemáticas y en estilos docentes, pero siempre entendiendo que una concepción (postura) supone el conocimiento de la materia. Modelos de concepción de la naturaleza de las matemáticas: dogmático-conservador, instrumentalista y pragmático-constructivista. Modelos en la concepción de las matemáticas: platónica, formalista e intuicionista. Clasificación de estilos docentes: tradicional, transitorio y avanzado

La investigación muestra los siguientes resultados: la concepción de las matemáticas predominante es platónico-formalista; y la práctica docente dogmática-conservadora, salvo en un caso que entiende debería ser pragmático-instrumentalista. Ningún profesor valora suficientemente a los estudiantes. Se considera a los alumnos receptores-pasivos, y que el aprendizaje se realiza por imitación; en ningún caso se estima la posibilidad de alumnos con diferentes estilos de aprendizaje, ni se piensa que se sentirían motivados mejorando el aprendizaje si la enseñanza se orientara a sus cualidades específicas. Es posible que el profesor no se preocupe por atender a variedades, porque esto le obligaría a cambiar la metodología de enseñanza para satisfacer las diferentes necesidades de aprendizaje y los diferentes niveles de estudiantes.

El fracaso se achaca a las actitudes de los estudiantes, y a su escasa formación. Por todo ello, el informe considera que el proceso de enseñanza se muestra: asimétrico, sin reparto de responsabilidad entre profesor y estudiante; falto de intencionalidad, pues metas y objetivos no quedan claros; la enseñanza se reduce a 
un conglomerado de contenidos conceptuales y tareas de ejercitación sin finalidad y se caracteriza por una excesiva idealización, pues el profesor acaba usando muchas ideas que no son comunicadas con claridad, ni compartidas con los estudiantes lo que afecta negativamente al proceso docente. Tanto el análisis general como el particular permiten sostener que el conocimiento de las creencias del profesorado sobre la docencia es fundamental, pues sólo sabiendo su consistencia, coherencia y permeabilidad es posible saber los factores que están determinando tanto el éxito como el fracaso en el proceso educativo, así como las posibilidades de su variación, pues cualquier intento de realización efectiva solo puede tener lugar si los profesores son conscientes de la necesidad de variación de su práctica.

El tercer trabajo es el realizado por Cristina Mayor Ruiz, en el Departamento de Didáctica y Organización Escolar y Métodos de Investigación y Diagnóstico en Educación de la Universidad de Sevilla, titulado Las funciones del profesor universitario analizadas por sus protagonistas. ${ }^{12}$ Sus objetivos son: 1. Indagar en los tres ámbitos representativos del profesor universitario: docencia, gestión e investigación; 2. Identificar los factores que limitan la labor docente, investigadora y de gestión, 3. Describir las diferencias profesionales en función de la especialidad académica; 4 . Apreciar la variedad de problemas y necesidades en profesores con experiencia y profesores principiantes; 5 . Buscar relaciones entre los distintos aspectos utilizando como técnica el análisis de correspondencias múltiples. Se trata de resolver estos objetivos en función de tres temas relevantes: dificultades en la enseñanza, cambios en la enseñanza, el factor tiempo y cambios en las condiciones profesionales. Para su evaluación se consideran básicos dos factores: antigüedad y especialidad disciplinar, al que se añade el género. El análisis fue realizado sobre 125 sujetos de las especialidades de: Ciencias, Humanas, Sociales y Sanitarias.

Tres titulaciones engloban el $91 \%$ del profesorado. La más numerosa es la de Ciencias 45\%, seguido de Humanas 32,2\%, Sanitarias 1,6\%. Pero, Sociales y Humanas pueden configurar un solo grupo que supondría el 45,8\% de la muestra. En antigüedad el $20 \%$ de la muestra no supera los tres años de antigüedad, mientras que el 33,6\% se encuentra entre 4 y 10 años de experiencia docente.

El instrumento utilizado es el Inventario sobre situaciones profesionales del profesor universitario (ISPPU), instrumento adaptado del propuesto por Lovett y otros, ${ }^{13}$ que la autora ha simplificado en función de las exigencias

\footnotetext{
12 Puede consultarse en: Revista electrónica de investigación y evaluación educativa// 1996//Volumen 2// Número 1. ISSN 1134-4032 // D.L. SE-1138-94

${ }^{13}$ Lovett, C. M. y otros. (1984). "Vitality without mobility: The Faculty opportunities audit". En: American Association for Higher Education, pp. 4, 2-43.
} 
validándose dos veces con las denominaciones Lovett 3 y Lovett 4 . Se explica la estructura del instrumento: partes de que consta, forma de presentación, items en que se desarrollan las funciones del profesor universitario, dimensiones ((docente e investigador (6 dimensiones), gestión (cinco dimensiones), ámbito profesional/formativo (cuatro dimensiones)). El cuestionario consta de 59 preguntas en total. En docencia (enseñanza-aprendizaje) incluye: planificación, metodología, tutorías, relación profesor-alumno, ambiente. En investigación incluye: resultados de investigaciones o producciones artísticas. En gestión incorpora un apartado de participación o colaboración con el departamento. A los tres ámbitos incorpora la dimensión: "limitaciones", es decir, factores que dificultan ejecución de las tareas examinadas.

En investigación se da cuenta de las fórmulas que presenta el inventario: respuestas de elección, de ordenación, abierta (libre). También se expone la técnica de análisis de datos utilizada, de la que se destaca su generalidad y versatilidad: el Análisis de Correspondencias Múltiples que se encuentra publicado en: Cornejo, J. M. (1988). Técnicas de investigación social: el análisis de correspondencias (teoría y práctica). Barcelona: PPU. Es una modalidad del Análisis de Correspondencias, que se considera en función de su posibilidad para analizar una población de individuos descrita por un conjunto de variables cualitativas, ordenarla matricialmente y tratar escalarmente tanto individuos como modalidades de variables. Por ello, la autora entiende que esta técnica permite solucionar el problema de la cuantificación de lo cualitativo, y que además tiene capacidad para representar en una estructura geométrica las relaciones multiespaciales entre individuos y variables. El tratamiento informático de los datos se realiza mediante el paquete BMDP Statistical Software, cuyos datos se concretan.

Las especialidades que agrupan a los profesores encuestados son: Humanas, Sociales, Ciencias, Técnicas y Sanitarias. Los problemas estudiados son: dificultades en la enseñanza, cambios en la enseñanza, tiempo dedicado a docencia, investigación, gestión, cambios en las condiciones profesionales.

Los resultados que ofrece la investigación son: los profesores de HUMANAS son sujetos con experiencia docente que no supera los 20 años de media. Emplean más tiempo en investigación. Señalan la necesidad de cambios en la profesión docente. Los profesores de SOCIALES dedican mayor tiempo a la planificación de la enseñanza. No proponen cambios en la profesión. Exhaustiva dedicación a la preparación de las clases. Poca atención a la investigación y a preocupaciones por reformas. Los profesores de CIENCIAS con experiencia docente entre 20 y 30 años, otorgan un papel relevante a la 
docencia, entienden que han aprendido a enseñar por experiencia. Los profesores de TÉCNICAS son los más experimentados; como docentes universitarios, entienden que el estilo docente no depende del estudio. No dedican casi tiempo a la investigación. Se inclinan por cambiar de profesión o de departamento. Los profesores de SANITARIAS presentan mucha dedicación a la enseñanza, pero escasa dedicación a la investigación. Los profesores más renovadores pertenecen a las titulaciones de Humanas y Técnicas, son profesores de los extremos, los más noveles y los más veteranos; los de Sociales se dedican más a la enseñanza.

\section{CONCLUSIONES}

\section{La necesidad de métodos de organización del conocimiento}

En las tres investigaciones se valora la calidad de forma fundamental en tres campos: docencia, gestión e investigación, relativamente a los que es posible saber como entender la experiencia de los profesores sobre los problemas que afectan a la relación enseñanza-aprendizaje. En el primer trabajo se considera la noción de creencia en función del factor autoeficacia, y se considera que su valoración depende, a su vez de tres factores: la preparación recibida, la responsabilidad de las realizaciones y la satisfacción. Se pone de relieve que la antigüedad (años de experiencia) es determinante de la variación de las creencias en general, en las que luego hay que delimitar los elementos propiamente psicológicos, de aquellos que pueden tener valor psicométrico y que permite alcanzar un nivel fiable de objetivación de experiencias. En la segunda investigación se distingue expresamente entre creencias (psicológicas) que se aplica al proceso de enseñanza-aprendizaje (metodología y organización de conocimientos), y concepciones que suponen conocimientos internos a la materia. El tercer trabajo no explicita claramente el término creencias, sino que lo recoge en la forma de experiencias cualitativas que se muestran en las respuestas abiertas, siendo éstas aquellas en las que el profesor puede expresarse libremente sobre el tema, y no simplemente marcar una casilla. Sí se distingue entre principiante y experto, es decir, profesional con mayor o menor antigüedad, y se intenta mostrar cómo entender la satisfacción del profesor en su profesión, las limitaciones que el desarrollo de su profesión le produce, así como el tiempo que dedica a cada campo

En la segunda investigación, se indica que las respuestas cualitativas, aquellas en las que se muestran elementos emocionales, afectivos, etc, (creencias), tienen que ser consideradas psicológicas, pero que tendrán que atenderse a ellas como datos significativos para apreciar los valores educativos en que los 
docentes cifran sus creencias respecto a la concepción de su materia. Un ejemplo puede ser el siguiente:

Pregunta: ¿La formación de los profesores como matemáticos está muy alejada de las aplicaciones a otros campos de las ciencias experimentales?

Respuesta: ¡Hombre claro que si! La mayor limitación no está en que puedes buscar ejemplos, ni en esto, sino que los ejemplos que buscas, ni el químico te los ha pedido porque no le interesa nada, o sea, ni tú, a lo mejor, los has buscado adecuadamente. Comprende, es que es una cosa que nadie te la ha pedido y además, no la necesitan; o sea, es que realmente... la sensación que tienes es que no te la han pedido y no la necesitan, iya está!

La respuesta comporta un problema: tenía que obedecer a una concepción, al conocimiento de un experto en la materia, encuadrarse en un modelo y dar paso a una cuantificación, pero expresa simplemente una creencia (según la definición dada) basada en una condición psico-física: tener la sensación. La pregunta exige que la respuesta comporte conocimiento en la materia; la respuesta no lo comporta, sino que el profesor expresa una valoración personal del problema, lo traslada del campo de las matemáticas (profesional) al vivido en las relaciones inter-profesionales; por lo que la respuesta tiene que considerarse una expresión cualitativa.

En el tercer trabajo se señala que las respuestas cualitativas, aportadas sobre todo en las respuestas abiertas, son tratadas mediante el análisis de correspondencias múltiples, que permiten ordenar escalarmente las descripciones en las que aquellas consisten.

En los tres trabajos se muestra la creencia, como noción conformadora de la experiencia en su aspecto cualitativo, y en ninguno es tomada globalmente, sino referida a campos precisos en los que las respuestas pueden adquirir valor concreto. La articulación y entre creencia y conocimiento (concepción), en la segunda investigación, que además constituye un caso relevante en cuanto afecta a la enseñanza de una materia fundamental, pero problemática, pues en ella se produce un alto fracaso, es muy importante para conocer las características en que la experiencia docente del profesor universitario cifra la enseñanza, estas son : pasividad, imitación y memoria, y hay que poner de relieve que el trabajo indica expresamente que los profesores carecen de conocimientos sobre el proceso enseñanza-aprendizaje. Este es un hecho en la enseñanza actual, y es necesario tenerlo en cuenta como un dato fundamental, en cuanto las tres investigaciones coinciden en señalarlo: el profesorado no 
tiene conocimientos sobre el proceso docente, y este conocimiento es condición necesaria para que aquél sea satisfactorio. En el caso de la creencia en la autoeficacia, se estima que indudablemente el conocimiento del profesorado de las distintas formas de autoeficacia y su valoración, consolidaría muy considerablemente el proceso docente.

En el caso de la enseñanza de las matemáticas, se estima que la transformación de creencias en concepciones, en lo que afecta al proceso enseñanza-aprendizaje, variaría muy favorable y considerablemente la relación profesor-alumno, afectando decisivamente a los estilos de enseñanza e incluso a la forma en que se percibe el nivel a alcanzar por los alumnos, pues la idea general del profesor actualmente es que se trata de introducir al alumno en el conocimiento de las materias (en este caso de las matemáticas), como si los alumnos fueran a ser profesionales de ellas.

De forma manifiesta, los tres trabajos coinciden en que la experiencia del profesor universitario manifiesta solo creencias y no conocimientos en lo que hace relación al proceso docente, y consideran que el paso de creencia a conocimiento, es un factor decisivo para una toma de conciencia de qué es necesario para que la enseñanza sea satisfactoria. En la búsqueda de éste conocimiento se hace hincapié en la necesidad de que se lleven a cabo relaciones interdisciplinares e interdepartamentales que, por una parte, favorezcan la percepción de su situación profesional por los profesores (ésta incide muy considerablemente en el rendimiento y éxito del proceso) y, por otra, favorezcan la cooperación entre especialistas de las distintas materias implicadas que lleve a saber qué tipo de concepción de la materia es la más adecuada, qué aplicaciones requieren la mejor orientación y preparación del alumno, y que lleve a producir un funcionamiento que posibilite alcanzar formas de sinergia.

El resultado del análisis y comparación de las tres investigaciones objeto de estudio, es la necesidad de lograr un marco común que permita considerar la forma de articular e integrar las experiencias cuantitativas y cualitativas. Este marco común, de hecho, es perfectamente delimitable en tanto los tres trabajos coinciden en destacar que: la experiencia docente del profesor universitario manifiesta una carencia total de conocimientos de organización del conocimiento, y que toda variación en el proceso de enseñanza-aprendizaje exige que el profesor adquiera esta capacidad como forma de llegar a la conciencia (en cada caso) de la realidad que se está produciendo en aquél. Por ello, la conclusión efectiva tiene que ser que intervenir en la práctica del profesor supone variar sus creencias, y esto requiere que el profesor adquiera capacidad de llegar a una conciencia sobre la realidad que está produciendo. Por tanto, 
intervenir en la experiencia del profesor requiere que éste adquiera conocimientos sobre métodos organización del conocimiento.

En conformidad con los análisis realizados, considero que la incidencia positiva de los tres trabajos sobre la calidad en el proceso docente supone partir de la toma de conciencia de que en la relación docente (profesor-alumno) no media un conocimiento objetivo a-priori (dado), sino que el profesor tiene una concepción cuya realidad como conocimiento objetivo tiene que comunicar y transmitir al alumno, que no tiene conocimiento el nivel suficiente de la materia. El reconocimiento de ésta situación, exige atender a las siguientes dos propuestas:

1.Que se posibilite la formación en métodos organización del conocimiento del profesor de educación superior, de manera que perciba las dificultades de objetivación de (su) conocimiento y concepción.

2.Que se posibiliten relaciones interdisciplinares e interdepartamentales.

Evidentemente esto en ningún caso supone que no exista un ámbito cultural común en el proceso docente mediador entre el alumno y el profesor, lo que supone este éste ámbito no está objetivado sin más, sino que es constituido y transformado en el mismo proceso-docente, por lo que exige su explicitación organizada y consciente.

\section{FUENTES}

PIAGET, J. y otros (1967): Logique et connaissance scientifique. Paris, Gallimard.

PIAGET, J. (1969): Las nociones de estructura y génesis. Buenos Aires, Proteo.

PIAGET, J. (1969): Biología y conocimiento. Madrid, Siglo XXI.

PIAGET, J. (1970): Le structuralisme. Paris, P.U.F.

POPPER, K. y ECCLES, J. (1985): El yo y su cerebro. Barcelona, Labor.

POPPER, K. (1985): La lógica de la investigación científica. Madrid, Tecnos.

POPPER, K. (1994): Conjeturas y refutaciones: el desarrollo del conocimiento científico. Barcelona, Paidós.

POPPER, K. (1998): Los dos problemas fundamentales de la epistemología. Madrid, Tecnos.

POPPER, K. (2005): Conocimiento objetivo: un enfoque evolucionista. Madrid, Tecnos.

POPPER, K. (2005): El mito del marco común: en defensa de la ciencia y la racionalidad. Barcelona, Paidós.

PRIETO NAVARRO, L. (2007): "Las creencias de autoeficacia docente del profesorado universitario", en: www.des.emerg.edu/mfp/PrietoSintesis.pdf.

Rev. hist. edu. latinoam. Vol. 15. Año 2010, pp. 177 - 196

\begin{tabular}{l|l} 
& Rev. hist. edu. latinoam. Vol. 15. Año 2010, pp. $177-196$ \\
\hline &
\end{tabular}




\section{REFERENCIAS}

AUSUBEL, D., NOVAK, J., HANESIAN, H. (1993): Psicología Educativa: Un punto de vista cognoscitivo. México: Trillas. (1978). Versión castellana de: (1978) Educational Psychology: A Cognitive View (2nd Ed.). New York, Holt Rinehart \& Winston.

BUNGE, M. (2002): Epistemología. Curso de actualización. $3^{\circ}$ ed. Barcelona, Ariel.

BUNGE, M. (2004): Emergencia y convergencia. Novedad cualitativa y unidad del conocimiento. Barcelona: Gedisa. Versión castellana de: (2003) Emergence and Convergence. Qualitative Novelty and the Unity of Knowledge. Toronto, University of Toronto Press.

BUNGE, M. (2008): Tratado de filosofía. Vol. I, Semántica 1: Sentido y referencia. Barcelona: Gedisa. Versión castellana de: (1974) Treatise on Basic Philosophy. Vol. I, Semantics 1: Sense and Reference. Dordrecht, Reidel.

BUNGE, M. (2009): Tratado de filosofía. Vol. II, Semántica 2: Interpretación y verdad. Barcelona: Editorial Gedisa. Versión castellana de: (1974): Treatise on Basic Philosophy. Vol. II, Semantics 2: Interpretation and Truth. Dordrecht, Reidel.

GEWERE, A. (2001): "Identidad profesional y trayectoria en la universidad", en: Profesorado. Revista de Vitae y Formación de Profesorado. Año/vol. 5. n ${ }^{\circ}$ 002. Granada, Universidad de Granada.

IBERMON, F. (2007): La formación permanente del profesorado. Barcelona, Grao.

LAFUENTE: M. I. (1977): Causalidad y conocimiento según Piaget. León: Colegio Universitario de León. Fundación de la Caja de Ahorros y Monte de Piedad de León.

LARROSA, J. (2007): “Algunas notas sobre la experiencia y sus lenguajes”, en: www.mc.gov.ar/ cumform/publica/oei/oei_20031128/potencia-larrosa.pdf.

LOVETT, C. M. y otros. (1984): "Vitality without mobility: The Faculty opportunities audit," en: American Association for Higher Education.

MAYOR RUIZ, C. (2007): "Las funciones del profesor universitario analizadas por sus protagonistas", en: Revista electrónica de investigación y evaluación educativa//1996//Nolumen 2// Número 1, D.L.SE.

MORENO, M. Y MORENO, C. (2007): “Concepciones y creencias de los profesores universitarios de matemáticas acerca de la enseñanza de las ecuaciones diferenciales”, en: Enseñanza de las ciencias. Vol. $21, \mathrm{n}^{\circ} 2$.

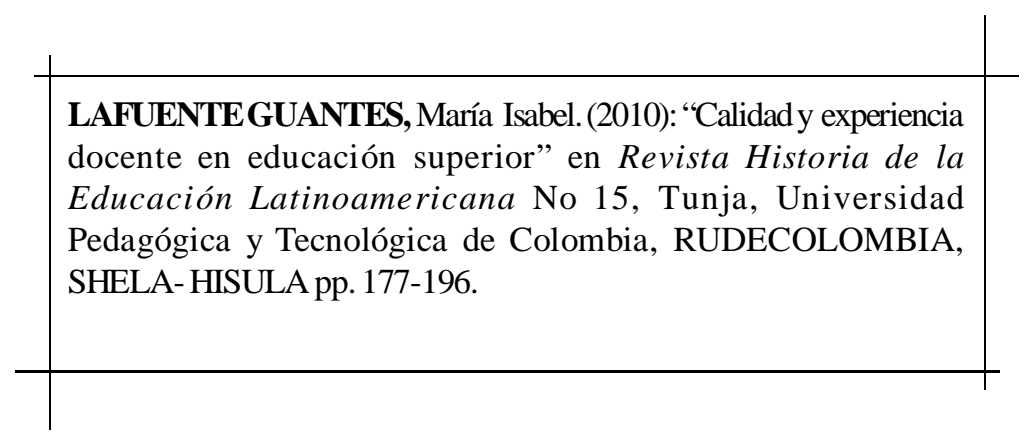

Rev. hist. edu. latinoam. Vol. 15. Año 2010, pp. 177 - 196 